\title{
Subsurface Geobody Imaging Using CMY Color Blending with Seismic Attributes
}

\author{
Jianhua Cao, Xiankun Zhang, Yan Wang, and Qi Zhao \\ College of Computer Science and Information Engineering, Tianjin University of Science and Technology, Tianjin 300222, China \\ Correspondence should be addressed to Jianhua Cao; caojh@tust.edu.cn
}

Received 3 November 2015; Revised 26 January 2016; Accepted 9 March 2016

Academic Editor: Sook Yoon

Copyright (C) 2016 Jianhua Cao et al. This is an open access article distributed under the Creative Commons Attribution License, which permits unrestricted use, distribution, and reproduction in any medium, provided the original work is properly cited.

Recently, ideas of color blending have brought the enlightenment for subsurface geobody imaging in petroleum engineering. In this paper, we present this approach of CMY color blending and its application in subsurface geobody characterization by using seismic attributes data. The first step is to calculate three types of seismic attributes based on the Hilbert transform algorithm, including envelop, instantaneous phase, and instantaneous frequency. Then scale the three attributes and combine them together using CMY color model in three-dimensional environment, with each attribute corresponding to one primary color channel. Adjust the scale and offset for each color component and then mix them optimally to create one color-blended volume. The blended volume in CMY mode has plenty of geological information coming from the three input attributes, resulting in high resolution and accurate image for subsurface geobodies. Applications show good performances in buried channels, caves, and faults imaging. Based on the blended slice, the geological targets can be easily but accurately interpreted and depicted.

\section{Introduction}

In petroleum engineering, subsurface geological targets, including channels, deltas, faults and carbonate caves, and reef, are very important factors for the researchers to find the oil/gas reservoir.

Geophysical prospecting is the conventional and reliable way for the oil/gas exploration. Among the geophysical tools, seismic data is the basic and critical data used to recognize shapes and location of sedimentary geological targets buried hundreds or thousands meters underground. Just like the modern geological phenomena on land, ancient deposits are complicated and difficult to be recognized. They might give no prominent response in seismic profiles. Data mining technique [1] is then to be used in seismic data interpretation. Information is extracted from seismic data and reorganized, which is then defined as the corresponding seismic attributes. Analysis and interpretation works [2-6] show that these attribute applications have satisfactory performances in geological target imaging and prediction.

In seismic attribute applications, three-dimensional visualization has been a core means for interpreters so that the geological information could be visualized in multiple angles.
Within the visualization process, color is the critical and powerful means used to get better visual elaborations $[7,8]$. When inputting the attribute volume into the $3 \mathrm{D}$ environment, one pixel in 3D space corresponds to one sample of the attribute volume. And the original attribute value is represented by color levels. So when adjusting the colorbar, we can get different display status of the attribute volume. There also is another type of display status, namely, the volumetric perspective rendering, and spatial 3D shapes for the geological objects can be delineated.

In practical analysis, interpreters seldom use just one single seismic attribute in reservoir interpretation and comprehensive research. Since different types of attributes have meaningful geological information from different views, it is necessary to consider them together based on the geological objects. Multiattribute combination analysis could yield more details and provide more contributions for geological objects delineation, and covisualization is just one kind of attributes fusion technique.

When covisualizing the attribute volumes, color blending techniques based on the color models are often recommended. RGB (red, green, and blue) model is the mostly used 


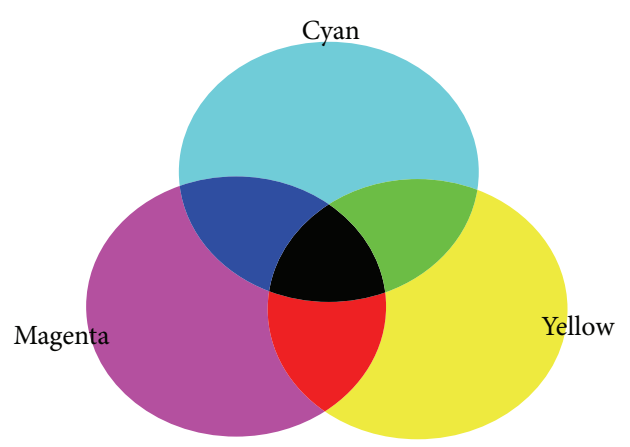

(a)

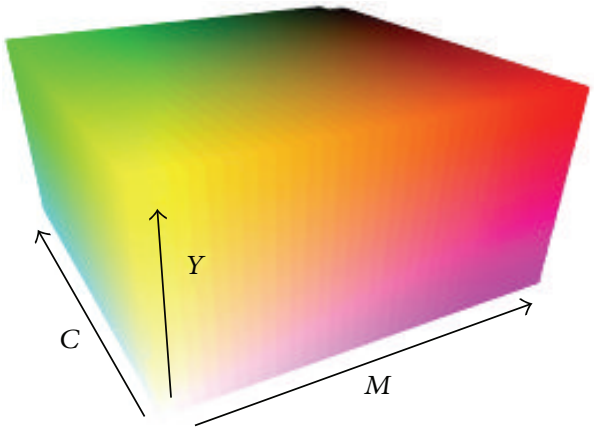

(b)

Figure 1: (a) Subtractive CMY model; (b) CMY color space.

in color blending, and it has been used in [9-11] to delineate geological elements using three types of seismic attributes.

In this paper, approach of color blending visualization in CMY mode using multiple seismic attributes is discussed for subsurface geobody imaging. Three basic attributes extracted from seismic data using Hilbert transform algorithm are used for color fusion. The attributes include envelop, instantaneous frequency, and instantaneous phase. When covisualizing in CMY mode, set envelop as the cyan component, instantaneous frequency as magenta component, and instantaneous phase as yellow component. And each component has its own scale and offset, so that the contributions of attributes could be manually adjusted and optimal blended result could be obtained finally. Horizontal slices are generated from the blended volume, and the geological targets can be recognized and interpreted by searching the specially lighted color zone on the CMY-blended slices.

The outline of this paper is organized as follows. In Section 2, the authors provide the methodology discussion, including the CMY color model, Hilbert transformation and seismic attributes, and color blending processing with the input attributes. The workflow of the multiattribute color blending is also presented in Section 2. Section 3 is devoted to describing the performances of the CMY color blending approach in several cases of geobody imaging. Finally, the paper is concluded in Section 4.

\section{Methodology}

2.1. CMY Color Models. Color is the visual perceptual property corresponding in humans to the categories called red, blue, yellow, and so forth. Color derives from the spectrum of light interacting in the eye with the spectral sensitivities of the light receptors [12]. In natural world, there are commonly several kinds of colors from human eyes, including red, blue, green, yellow, orange, and so forth. Some colors are primary, and some colors are formed by intermixing primary colors. Color also has its own physical properties, including hue, saturation, brightness, and gloss.
A color model [13] is an abstract mathematical model describing the way colors can be represented as tuples of numbers, typically as three or four values or color components. When this model is associated with a precise description of how the components are to be interpreted, the resulting set of colors is called color space. Red, blue, and green colors are defined as primaries, and when intermixing two or three primary colors, we can get thousands of colors. Naturally, there are two basic color models: RGB model and CMY model. RGB model is additive model using the three primary colors, including red, green, and blue. References [14-16] have discussed the detailed definition and introduction about RGB model. And, in this paper, we just focus on the CMY model.

CMY model is subtractive color model using primary colors of cyan, magenta, and yellow [17]. These three components are the complementary colors of red, green, and blue, respectively. Cyan originates from mixing blue and green, and magenta is the mixture outcome of red and blue. When mixing red and green, they produce yellow color. Considering the CMY mode, the final mixture depends on the contributions from the three original components. When all the intensity of three primaries are full, it produces black. When intensity of the magenta channel is zero and the other two are full, the mixture is green. And white is the natural color. The CMY model is shown as Figure 1(a) and the corresponding CMY color space as Figure 1(b). This model is mainly used in color printing. And it has advantages in depicting the edge of objects which may be favorable for the geological object imaging in $3 \mathrm{D}$ visualization environment.

2.2. Hilbert Transform and Seismic Attributes. Seismic attributes are the data or information mining from the seismic amplitude data, either by direct measurements or by logical or experience-based reasoning [18]. Effective seismic attributes can better understand and interpret the geological or geophysical properties of the subsurface deposits. By using seismic attributes, the vertical and lateral variations of the geological objects could be measured qualitatively and quantitatively. 


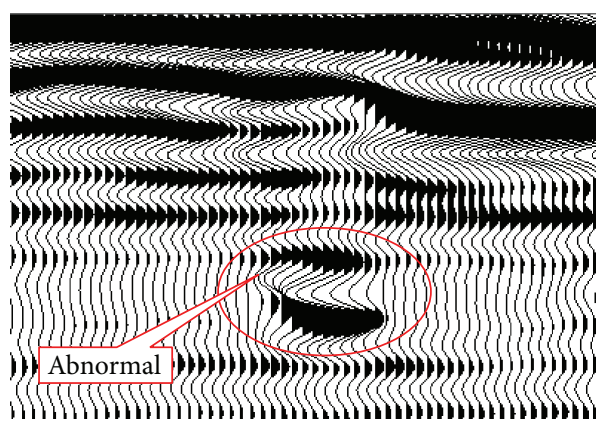

(a) Seismic signal

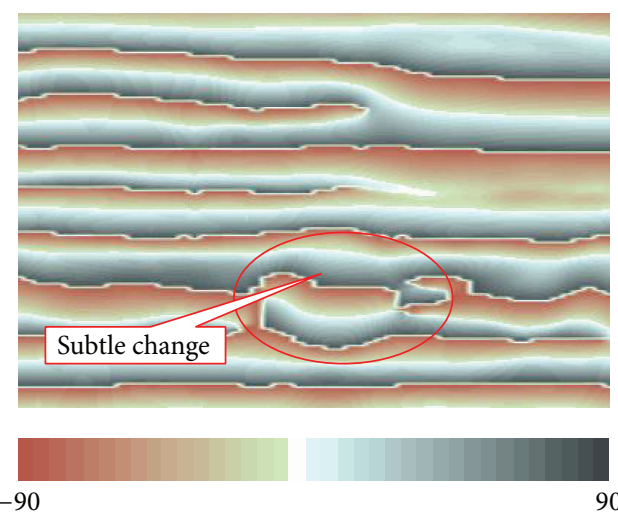

$\left({ }^{\circ}\right)$

(c) Instantaneous phase

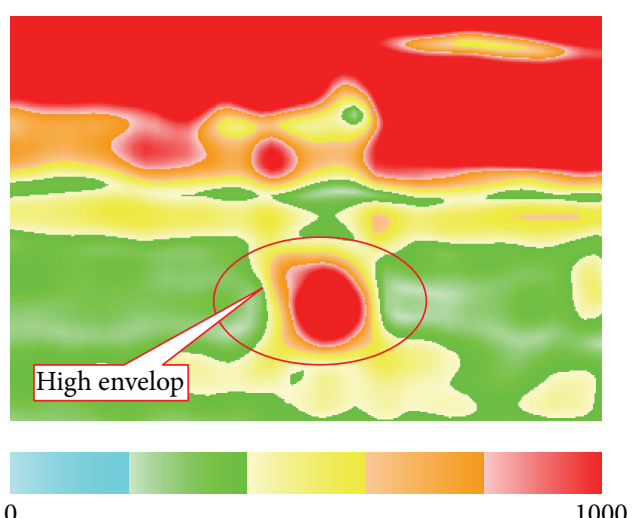

(b) Envelop attribute

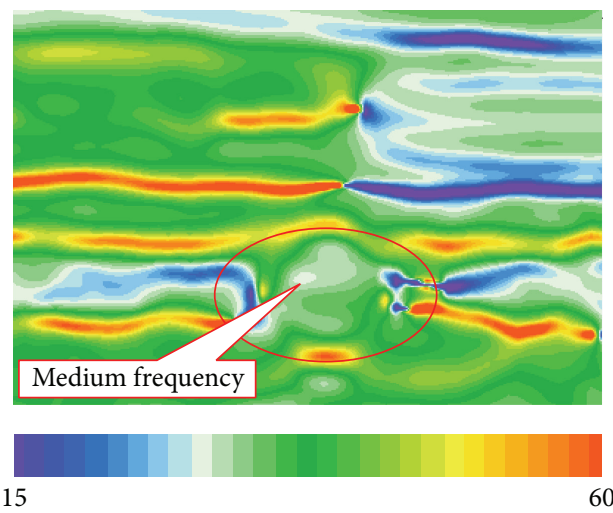

$(\mathrm{Hz})$

(d) Instantaneous frequency

Figure 2: The original seismic signal and its derivated attributes. The abnormal seismic waves show specific characters in the three attribute profiles, respectively.

The basic algorithm for the data mining technique is the Hilbert transform [18].

Given the seismic time-series data as $s(t), t \in R$, the Hilbert transformation $\widehat{u}(t)$ is expressed by

$$
\widehat{u}(t)=s(t) * h(t)=\int_{-\infty}^{\infty} s(\tau) h(t-\tau) d \tau,
$$

where

$$
h(t)=\frac{1}{\pi t}
$$

So the transform can be considered as the convolution of the $s(t)$ with the function $h(t)$, and the result of transform is

$$
\widehat{u}(t)=\frac{1}{\pi} \int_{-\infty}^{\infty} \frac{s(\tau)}{t-\tau} d \tau .
$$

Therefore the complex analytic signal $z(t)$ can be defined as

$$
z(t)=s(t)+j \widehat{u}(t)
$$

where $j=\sqrt{-1}$.
According to (4), several types of attributes representing the characters of seismic signal can be calculated. The primary attributes include envelop, instantaneous phase, and instantaneous frequency. Here we give the equations as follows.

For the envelop attribute $A(t)$,

$$
A(t)=\sqrt{s^{2}(t)+\widehat{u}^{2}(t)} .
$$

For the instantaneous phase $\phi(t)$, it is defined as

$$
\phi(t)=\tan ^{-1}\left[\frac{\widehat{u}(t)}{s(t)}\right] .
$$

For the instantaneous frequency $f(t)$, it is defined as

$$
f(t)=\frac{d \phi(t)}{2 \pi d t} .
$$

Figure 2 shows the original seismic data and the estimated typical attributes. The original seismic signals are wiggle display, and it is hard to depict the subtle changes except the obvious peak and trough reflection characters, while, on the attributes sections, the variations of color are much clearer, standing for lithological and physical property changes of subsurface geological objects. 


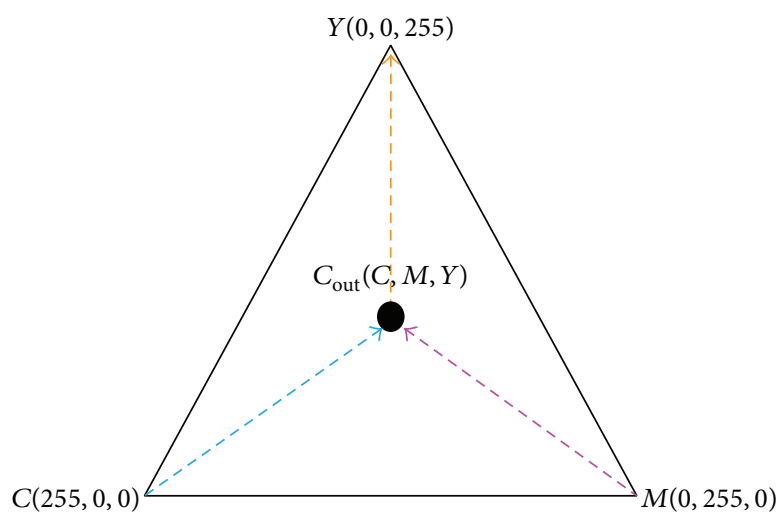

(a) CMY color blending

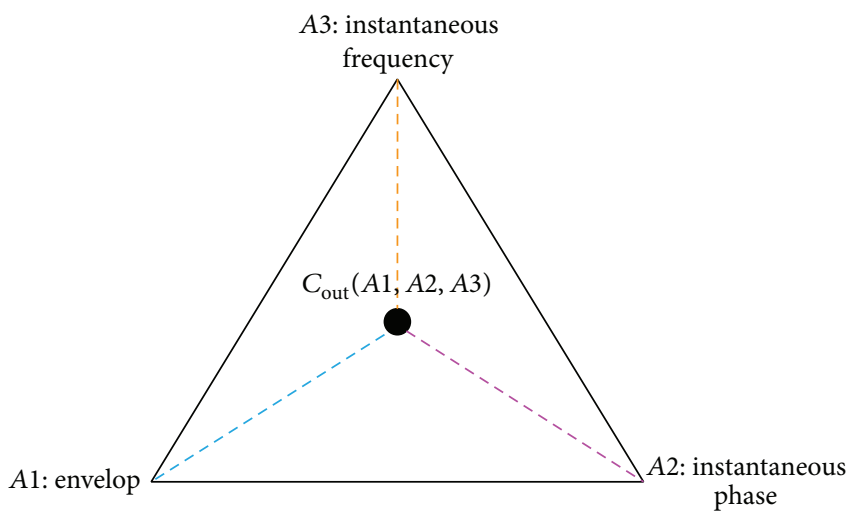

(b) Attributes blending

FIGURE 3: Diagram for CMY color blending and seismic attributes blending in CMY mode.

2.3. CMY Color Blending Using Seismic Attributes. Most of the typical visualization environments use just 8-bit color tables; thus, for each primary color in RGB mode, the values or the levels range from 0 to 255.0 stands for the darkest and 255 for the lightest. CMY color model has the same principle although its primary components are just from mixture of red, green, and blue.

Figure 1(b) shows the CMY color blending in threedimensional space. In the space, cyan is set as $x$-axis, and magenta color is set as $y$-axis, while yellow color is set as $z$ axis. For each pixel in 3D environment, its value for display is decided by levels of the three input components. The relations are just as follows:

$$
\text { Color of pixel }=\left(C_{l}, M_{l}, Y_{l}\right) \text {, }
$$

where $C_{l}$ stands for level of cyan, $M_{l}$ for level of magenta, and $Y_{l}$ for level of yellow.

If levels of the three inputs are all 255, the mixture color of pixel is $(255,255,255)$, and the subtractive result is black. And, in such model, green color can be expressed by $(255,0$, $255)$, red color by $(0,255,255)$, and blue color by $(255,255,0)$. Therefore, for the CMY model, the output blending results are just decided by the levels of the three input primary components.

In the mixing process, scale and offset can also be added to the components, respectively, which is

\section{Color of pixel}

$$
=\left(\left[\alpha_{1} C_{l}+\beta_{1}\right],\left[\alpha_{2} M_{l}+\beta_{2}\right],\left[\alpha_{3} Y_{l}+\beta_{3}\right]\right),
$$

where $\alpha_{i}, i=1,2,3$, is the scale or coefficient and $\beta_{i}, i=$ $1,2,3$, is the offset for the input color components in CMY blending process. And the ranges of the input primaries are limited in [0-255].

This type of blending technique can also be used for multiple-attributes fusion visualization in $3 \mathrm{D}$ seismic interpretation.

Three types of basic attributes are chosen to be the three primary color components, respectively. Before the fusion process, the normalization step is to be carried out so that the attributes become dimensionless. We linearly normalize the attributes to the same level range of primary color using the following formula:

$$
\text { Level of component }=256 * \frac{\left(\mathrm{Att}-\mathrm{Min}_{A}\right)}{\left(\operatorname{Max}_{A}-\operatorname{Min}_{A}\right)},
$$

where Att stands for the attribute value, Min for the minimum, and Max for the maximum value.

The CMY blending is then formed by using the three primary normalized attribute components. One attribute corresponds to one channel, respectively. So that for one pixel $(x, y, z)$ in the $3 \mathrm{D}$ color space, the color is defined by the values in the three input normalized images:

$$
\begin{aligned}
& C_{\text {out }}(x, y, z)=C\left(\left[\alpha_{1} I C(x, y, z)+\beta_{1}\right],\right. \\
& \left.\quad\left[\alpha_{2} I M(x, y, z)+\beta_{2}\right],\left[\alpha_{3} I Y(x, y, z)+\beta_{3}\right]\right),
\end{aligned}
$$

where $C_{\text {out }}(x, y, z)$ is the color assigned to the point $(x, y, z)$ in the output image and $\operatorname{IC}(x, y, z), \operatorname{IM}(x, y, z)$, and $I Y(x, y, z)$ are the pixel values at point $(x, y, z)$ in the input attribute images that are assigned to control the cyan, magenta, and yellow contributions, respectively. And $\alpha_{i}, i=$ $1,2,3$, is the scale or coefficient and $\beta_{i}, i=1,2,3$, is the offset for the input color components in blending process.

For better understanding the whole blending work, diagrams comparison for CMY blending and the corresponding attribute blending in CMY mode have been made as Figure 3.

2.4. Color Blending Workflow. We give the workflow for seismic attributes combination in 3D visualization space using CMY mode as Figure 4.

There are totally five steps. The first step is the seismic data conditioning. We usually use the poststack seismic data as the original input data. And there may be noise or abnormal values in it. We take some measures, including noise filtering and despiking, to remove them from the seismic data so that the data quality could be improved and might be ready for the next step. The second step is to carry out the Hilbert transform using the conditioned data and calculate three 


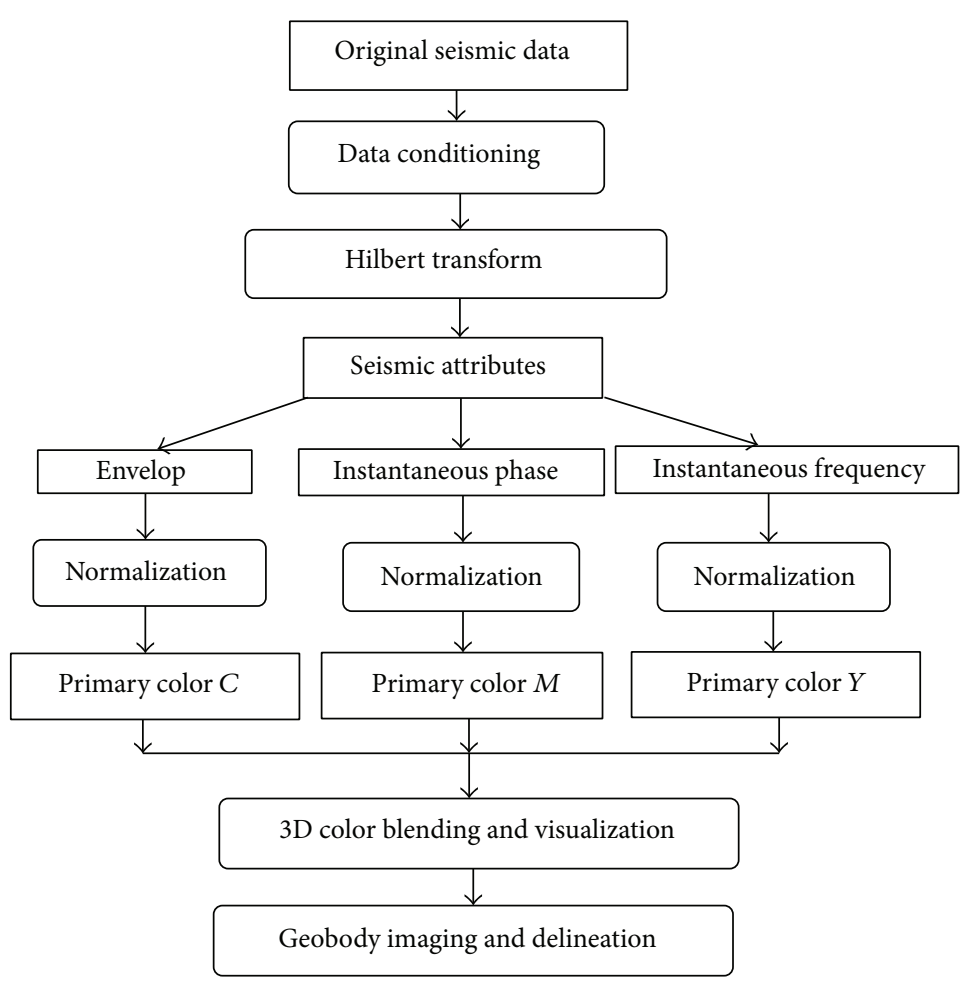

FIGURE 4: CMY color blending workflow for subsurface geobody imaging.

types of seismic attributes, which include envelop, instantaneous phase, and instantaneous frequency. When the three attributes are ready, the procedure goes to the color blending preparation step. The three types of attributes are to be normalized according to the color level ranging from 0 to 255 and then set the three attributes as the three input primary components, with one attribute corresponding to one primary color component. Then color blending process is performed in $3 \mathrm{D}$ visualization environment using the three primary components. The outcome is one CMY colorblended volume, which could be adjusted to be optimal using coefficients and offsets for each component. Finally, we focus on the analysis and interpretation using such color-blended and meaningful result. Of course, the whole interpretation process is executed based on the professional knowledge. Through browsing every horizontal slice near the target geobodies, we can find a lot of interesting color distribution characters. Some are just the images of our targets or edges of the geobodies. Rendering mode can be helpful in delineating the $3 \mathrm{D}$ shapes of the subsurface targets. By the way, we should mention that all the interpretation work relies on understanding the color distribution patterns on slices and the whole blended volume.

\section{Applications}

In this research, the proposed technique and workflow have been applied in several cases to characterize the geological targets, including the ancient channels, carbonate karst caves, and fault edges.
In case 1 , the workflow is sequentially carried out and subsurface buried channels are well imaged on the slices of the colored volume. To get better image, we adjust the coefficients and offsets of the input components after normalization. Figure 5(a) is the slice extracted from the colored volume. The abnormal dark and blue color belts are very attractive, which is just the response from subsurface channels. Based on the distinguishing character of colors, channels and their branches are very easy to be recognized. Figure 5(b) is the channel geological depiction based on the left color-blended slice. The main stream and the branches are very clear, and the surrounding plain is filled with muds overflowing from the channels. This is similar to the modern river on land.

Case 2 is another example of channel recognition using the blending workflow. Figure 6(a) is the slice from the blended volume. Notice the winding dark color belts in the middle area of the slice. They are just the buried channels. It is vividly shown and imaged. The shape character is the same as modern rivers (shown as Figure 6(b)).

Case 3 is an example of karst caves imaging using the three seismic attributes and the CMY blending workflow. Figure $7(\mathrm{a})$ is the slice from the blended volume. The karst caves are well imaged on the slice, and those highlighted irregular zones filled with blue and green color are the focus. The caves are always filled with low-velocity mudstone, which is different from the surrounding tight carbonate, so there always exists abnormal properties for the caves. In this case, the caves are imaged with blue and green color. Based on this, the caves can be geologically delineated horizontally (shown as Figure 7(b)). 


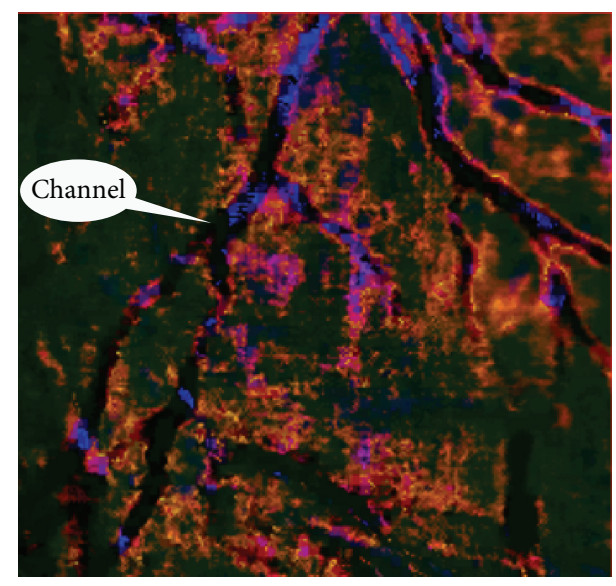

(a)

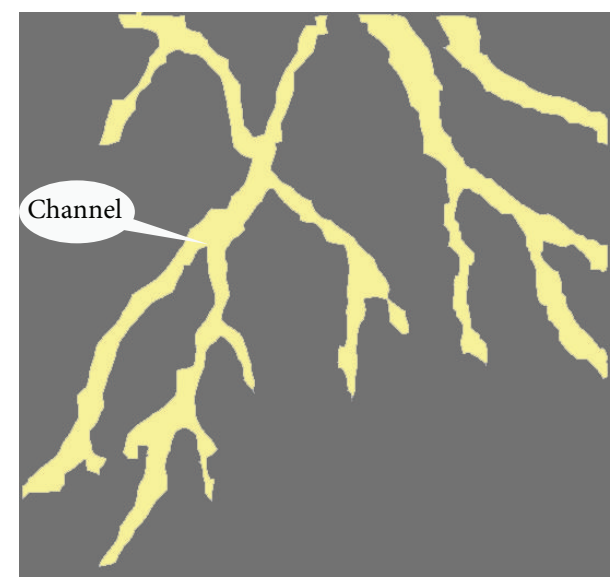

(b)

FIGURE 5: (a) Slice of the color-blended volume; (b) channel geological interpretation.

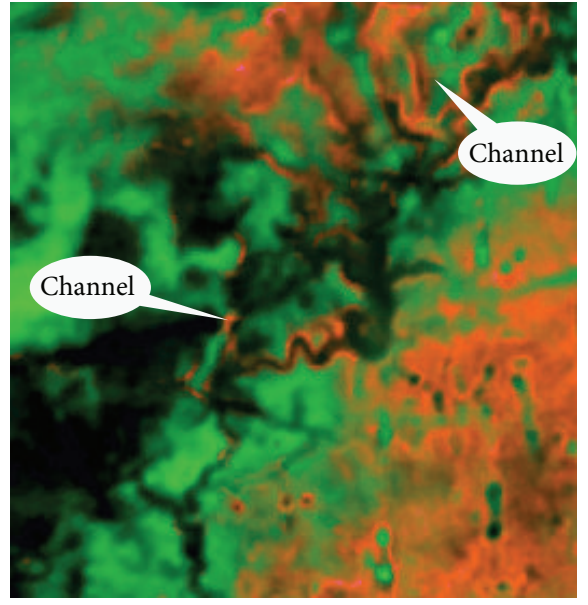

(a)

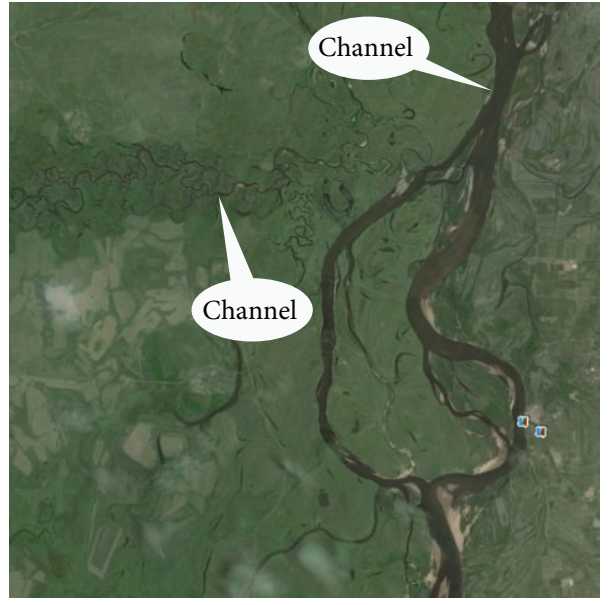

(b)

Figure 6: (a) Slice of the color-blended volume; (b) modern rivers on land (image snapped from Google Earth).

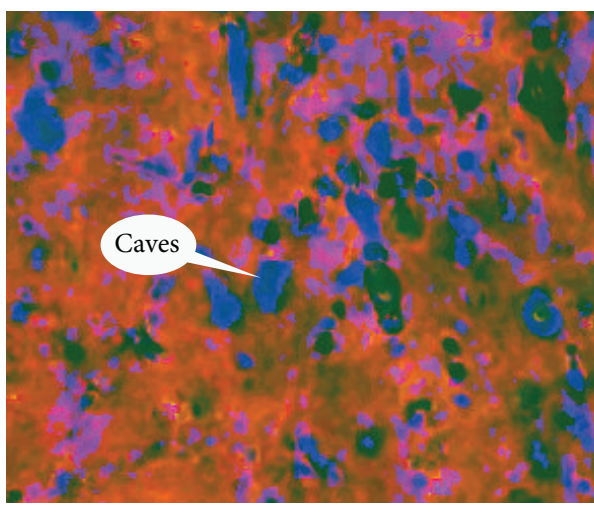

(a)

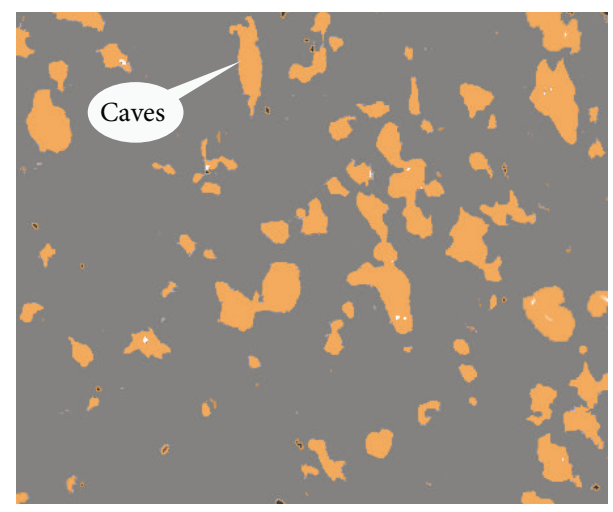

(b)

Figure 7: (a) Slice of the color-blended volume; (b) carbonate karst caves analysis based on the slice. 


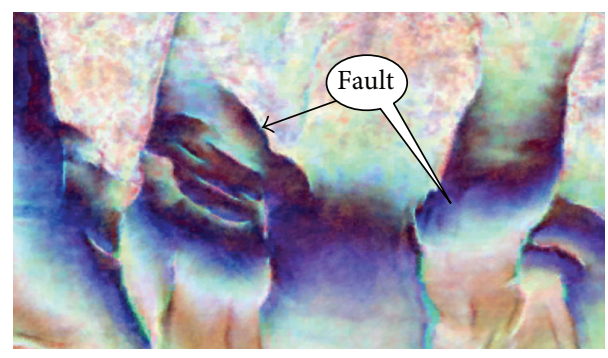

FIGURE 8: Slice of the color-blended volume.

Case 4 is an example of fault edge detection using CMY color mode blending technique. Figure 8 is the blended slice. The color mutation has its geological meaning. In this case, the boundary lines between different color zones are just the structural faults. And this type of images helps a lot for the structure interpreter and researchers.

\section{Conclusions}

For the subsurface geological targets, they are very difficult to be accurately imaged since they are invisible and measured directly using the traditional tools. In the paper, the authors discuss CMY color model and its application in the fusion of multiple seismic attributes for several types of targets imaging. The authors set up one effective workflow for attributes blending in CMY color model in $3 \mathrm{D}$ visualization space. Color-blended volume is generated by blending three types of basic seismic attributes, and slices of the blended volume are made and used to analyze and interpret the geological targets. Applications of the blended model in several cases show satisfactory performances. Shapes and distribution characters of subsurface targets, including channels, caves, and faults, have been vividly delineated based on the CMY-blended attribute slice. This kind of color mixed mode of thinking is very useful and effective in attribute fusion and object imaging and can also be recommended in prediction and analysis of other types of geological bodies in petroleum exploration.

\section{Competing Interests}

The authors declare that they have no competing interests.

\section{Acknowledgments}

This paper was supported by the Key Technologies R \& D Program of Tianjin no. 15ZCZDGX00200 and Tianjin Science and Technology Popularization Project no. 15KPXM01SF014.

\section{References}

[1] S. Chopra and K. J. Marfurt, "Seismic attributes-a historical perspective," Geophysics, vol. 70, no. 5, pp. 65-68, 2005.

[2] D. Ohl and A. Raef, "Rock formation characterization for carbon dioxide geosequestration: 3D seismic amplitude and coherency anomalies, and seismic petrophysical facies classification,
Wellington and Anson-Bates Fields, Kansas, USA," Journal of Applied Geophysics, vol. 103, pp. 221-231, 2014.

[3] J. W. Snedden, "Channel-body basal scours: observations from 3D seismic and importance for subsurface reservoir connectivity," Marine and Petroleum Geology, vol. 39, no. 1, pp. 150-163, 2013.

[4] C. G. Eichkitz, J. Amtmann, and M. G. Schreilechner, "Calculation of grey level co-occurrence matrix-based seismic attributes in three dimensions," Computers and Geosciences, vol. 60, no. 5, pp. 176-183, 2013.

[5] A. E. Raef, F. Mattern, C. Philip, and M. W. Totten, “3D seismic attributes and well-log facies analysis for prospect identification and evaluation: interpreted palaeoshoreline implications, Weirman Field, Kansas, USA," Journal of Petroleum Science and Engineering, vol. 133, pp. 40-51, 2015.

[6] P. Steeghs, I. Overeem, and S. Tigrek, "Seismic volume attribute analysis of the Cenozoic succession in the L08 block (Southern North Sea)," Global and Planetary Change, vol. 27, no. 1-4, pp. 245-262, 2000.

[7] B. Froner, S. J. Purves, J. Lowell, and J. Henderson, "Perception of visual information: the role of colour in seismic interpretation," The First Break, vol. 31, no. 4, pp. 29-34, 2013.

[8] A. E. Raef, T. N. Meek, and M. W. Totten, "Applications of 3D seismic attribute analysis in hydrocarbon prospect identification and evaluation: verification and validation based on fluvial palaeochannel cross-sectional geometry and sinuosity, Ness County, Kansas, USA," Marine and Petroleum Geology, vol. 73, pp. 21-35, 2016.

[9] D. Iacopini and R. W. H. Butler, "Imaging deformation in submarine thrust belts using seismic attributes," Earth and Planetary Science Letters, vol. 302, no. 3-4, pp. 414-422, 2011.

[10] Ó. Pueyo Anchuela, A. Luzón, H. Gil Garbi, A. Pérez, A. Pocoví Juan, and M. A. Soriano, "Combination of electromagnetic, geophysical methods and sedimentological studies for the development of 3D models in alluvial sediments affected by karst (Ebro Basin, NE Spain)," Journal of Applied Geophysics, vol. 102, no. 1, pp. 81-95, 2014.

[11] P. Kovesi, E.-J. Holden, and J. Wong, "Interactive multi-image blending for visualization and interpretation," Computers \& Geosciences, vol. 72, no. 4, pp. 147-155, 2014.

[12] http://en.wikipedia.org/wiki/Colour.html.

[13] L. Busin, N. Vandenbroucke, and L. Macaire, "Color spaces and image segmentation," Advances in Imaging and Electron Physics, vol. 151, pp. 65-168, 2008.

[14] J. Henderson, S. J. Purves, and F. Gaynor, "Delineation of geological elements from RGB color blending of seismic attribute volumes," The First Break, vol. 27, no. 3, pp. 21-26, 2008.

[15] B. Froner, J. Lowell, and S. J. Purves, "On the perception of RGB multi-attribute displays," PETEX, 2012, http://www.geoteric .com/uploads/downloads/PETEX_RGB_Displays.pdf.

[16] http://en.wikipedia.org/wiki/Additive_color.html.

[17] J. A. Farrell, "Color models and programming colors," in From Pixels to Animation, pp. 163-191, Elsevier, 1994.

[18] Rock Solid Images, http://www.rocksolidimages.com/attributesrevisited/. 


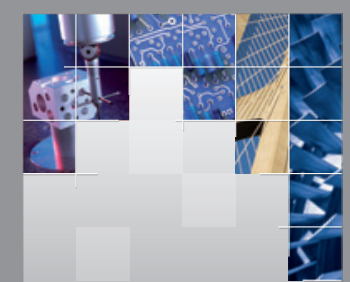

\section{Enfincering}
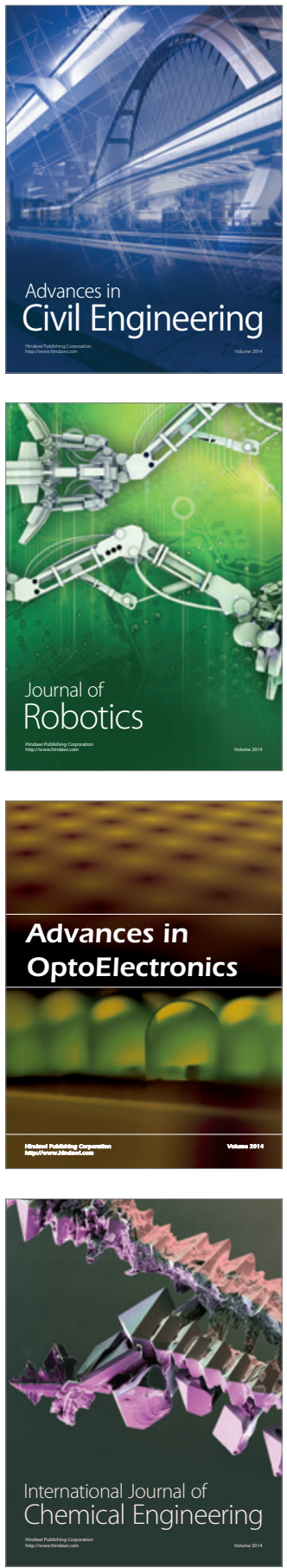

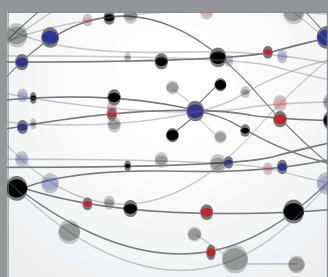

The Scientific World Journal

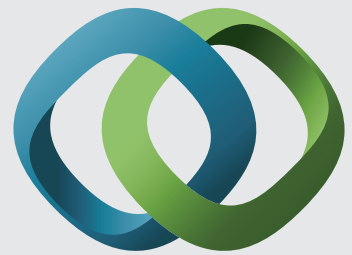

\section{Hindawi}

Submit your manuscripts at

http://www.hindawi.com
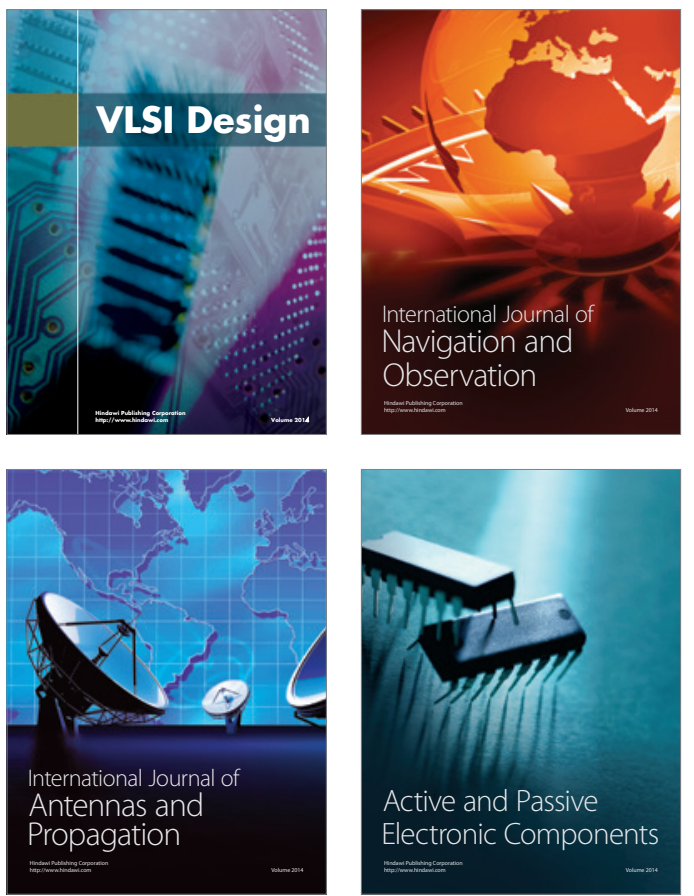
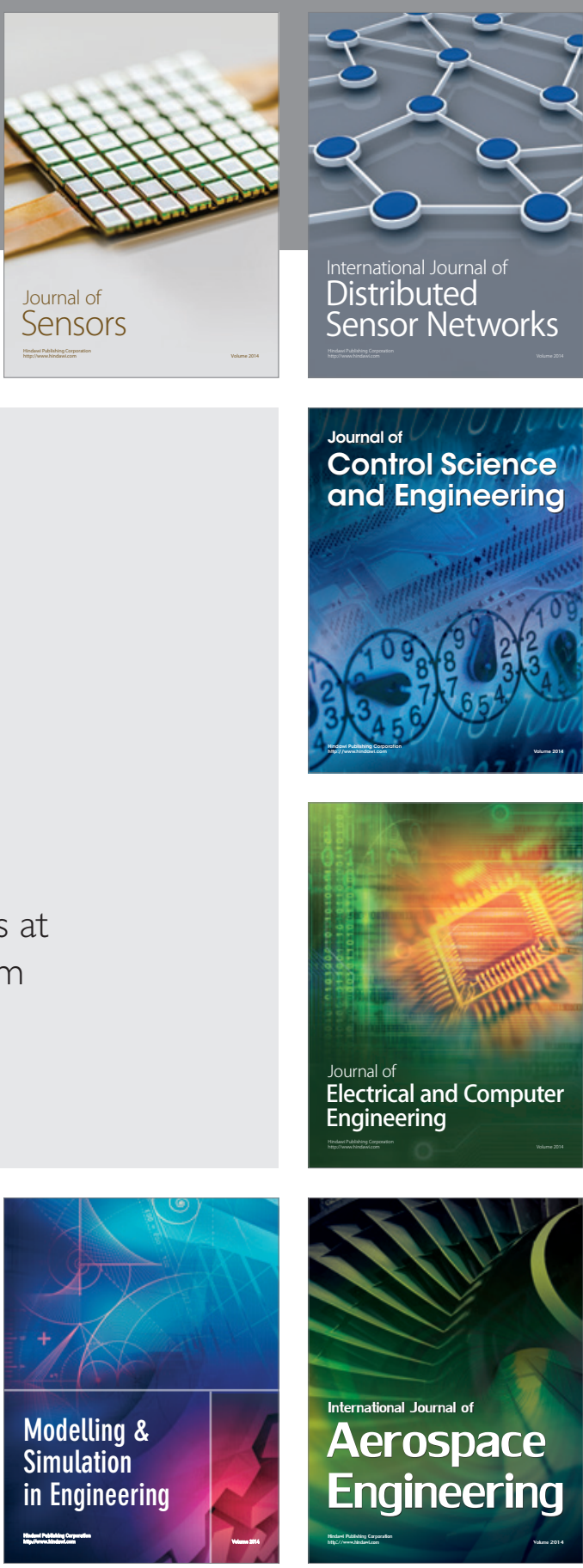

International Journal of

Distributed

Sensor Networks

Journal of

Control Science

and Engineering
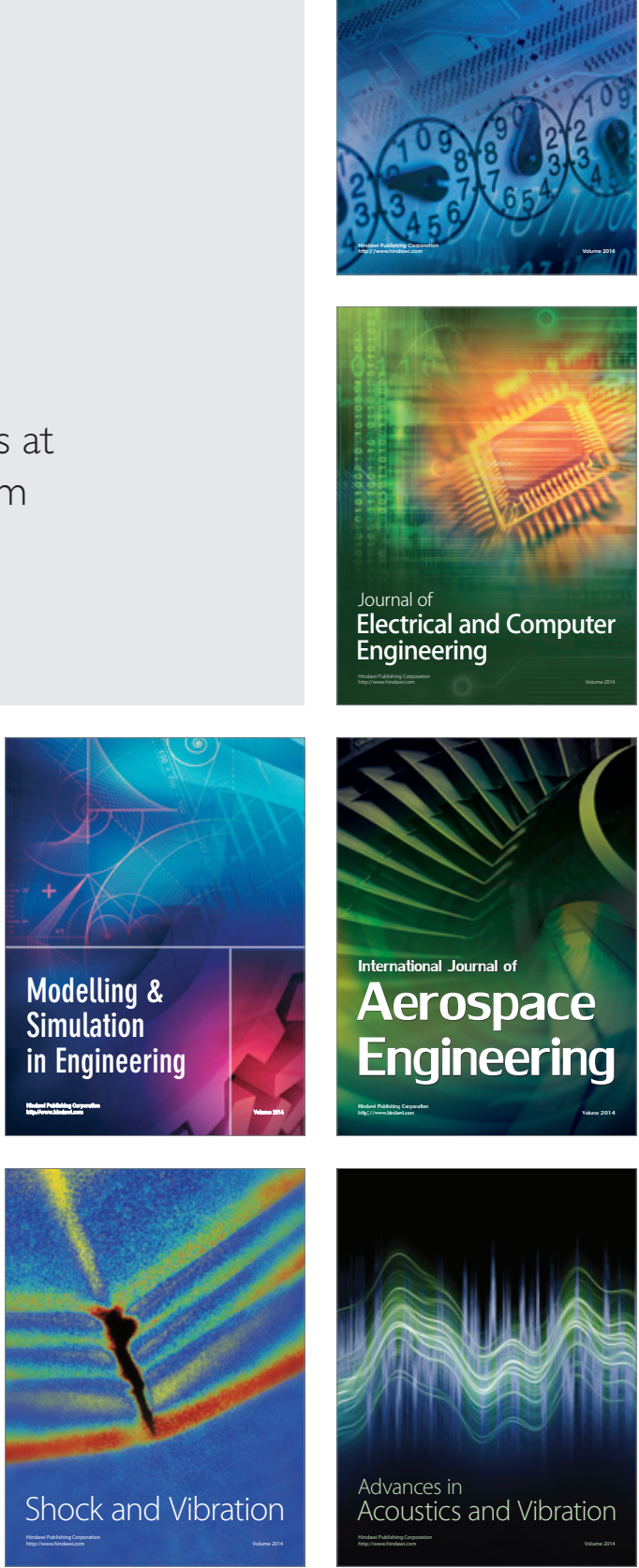